\title{
An Open Dynamical System Approach to Dissipative Quantum Dot Array Antennas
}

This paper was downloaded from TechRxiv (https://www.techrxiv.org).

LICENSE

CC BY 4.0

SUBMISSION DATE / POSTED DATE

04-01-2022 / 07-01-2022

CITATION

Mikki, Said (2022): An Open Dynamical System Approach to Dissipative Quantum Dot Array Antennas. TechRxiv. Preprint. https://doi.org/10.36227/techrxiv.17815241.v1

$\mathrm{DOI}$

10.36227/techrxiv.17815241.v1 


\title{
An Open Dynamical System Approach to Dissipative Quantum Dot Array Antennas
}

\author{
Said Mikki ${ }^{1}$
}

\begin{abstract}
We develop a general computational approach for the analysis and design of quantum antenna systems comprised of coupled quantum dot arrays interacting with external fields and producing quantum radiation. The method is based on using the GKSL master equation to model quantum dissipation and decoherence. The density operator of a coupled two-level quantum dot (qbit) array, excited by classical external signals with variable amplitude and phase, is evolved in time using a quantum Liouville-like equation (the master equation). We illustrate the method in a numerical example where it is shown that manipulating the phase excitations of individual quantum dots may significantly enhance the directive radiation properties of the quantum dot antenna system. ${ }^{1}$
\end{abstract}

Index Terms-Quantum antennas, quantum technologies, open quantum systems, master equations, directivity.

\section{INTRODUCTION}

Quantum antenna theory is an emerging new field within the larger area of quantum technologies [1], [2] and optical antennas [3], [4], itself part of quantum engineering [5]. The main objective of quantum antenna theory is to understand the fundamental principles of quantum antennas relative to classical antennas [6], [7]. Classical antennas are often used for applications mainly wireless communications [8], [9]. On the other hand, quantum antennas not only can support the transmitting and receiving terminals of quantum communications [10], [11], but moreover, due to the inherent richness of quantum radiation phenomena, a quantum antenna system can be used for a wide range of other applications such as bio-sensing, photonic energy devices, quantum imaging and tomography, and high-speed computing chip design [5], [12].

Quantum dots (QD) are now ubiquitous in nanotechnology, nanoelectronics, and quantum optics [3], [13]. A QD is essentially a 0 -dimensional quantum confinement system, acting like a resonator. It can be utilized in applications involving both fermions (e.g., electrons) and bosons (e.g., photons, plasmons). In this paper, we investigate deploying two-level QDs for building a quantum dot antenna (QDA) system capable of directing quantized electromagnetic radiation (bosonic photon field) toward specific directions (see Fig. 1.)

Anticipating the use of quantum dots and cavity QED methods borrowed from quantum optics [14] for the future task of realizing practical devices in quantum antenna technology, we propose a general computational framework for handling generic coupled arrays of QDs and radiation fields, where external control is permitted through electric-dipole mediated coupling with user-defined excitation fields, e.g., tailored laser

\footnotetext{
${ }^{1}$ The author is with the ZJU/UIUC Institute, China. He can be reached at said.m.mikki@gmail.com
}

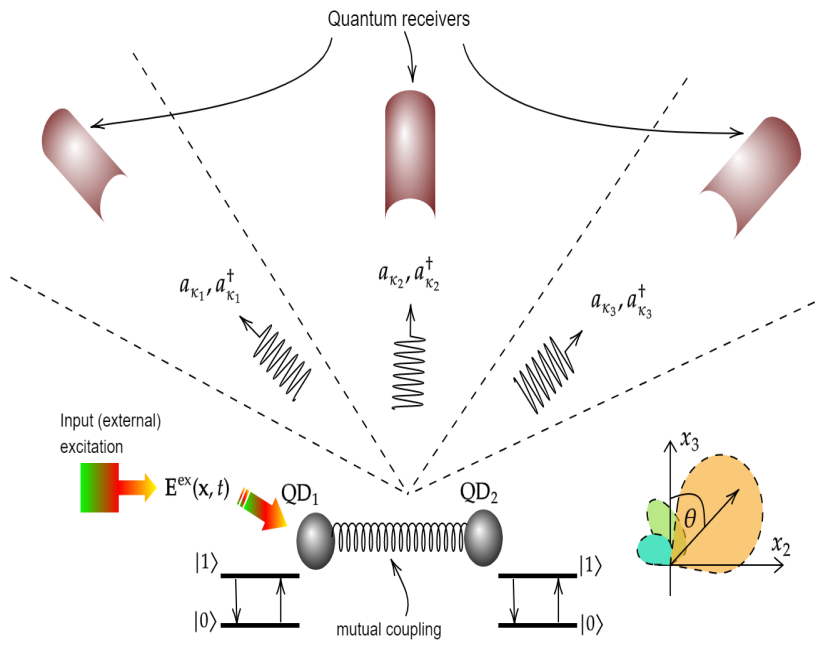

Fig. 1: The general configuration of the proposed quantum dot antenna (QDA) system.

pulses or microwave signals. Our analysis is based on adopting the viewpoint of quantum dynamical systems [15], also sometimes known as quantum stochastics [16], which treats open dynamical systems that are continuously exchanging energy/matter with their external environments while evolving in time [17]. This emphasis on open systems allows deeper insight into the dynamics of the various quantum processes, ability to model noise, and improving accuracy for future comparison with experiments. In what follows, we develop the basic ideas of the open dynamics approach, including modeling assumptions and the method's mathematical derivation. We propose a construction of the QDA directivity inspired by the corresponding expression in classical antenna theory. The algorithm is implemented and illustrated in a practical example of two-element QDA array system.

\section{Coupled QuAntum Dots And Radiation Fields: BASIC MODELING Assumptions}

The system under consideration include two main types of interacting quantum oscillators: 1) material quantum oscillators (quantum dots), which can be any multi-level quantum system; and 2) radiation quantum oscillators (quantized electromagnetic field), i.e., a bosonic system with infinite number of possible energy levels. In practice, we need to truncate the number of energy levels in order to perform realistic calculations in reasonable time. In the first approximation, we restrict the QDs to two-levels (this restriction will be 
relaxed in a future work). In the second approximation, only a finite (often) small number of bosonic energy levels will be considered.

Consider an QD array consisting of $M_{\mathrm{d}}$ dots, each positioned at $\mathbf{x}_{i}$. Associated with each QD is the raising and lowering operators $\sigma_{i}^{+}$and $\sigma_{i}^{-}$, respectively. Physically, the raising operator, when acting on a vacuum state, will produce an excited state: $\sigma_{i}^{+}\left|\varepsilon_{1}^{i}\right\rangle=\left|\varepsilon_{2}^{i}\right\rangle$, where $\varepsilon_{1}^{i}<\varepsilon_{2}^{i}$, while $\left|\varepsilon_{1}^{i}\right\rangle$ and $\left|\varepsilon_{2}^{i}\right\rangle$ are the ground and excited energy states of the $i$ th two-level QD (qbit) system, respectively [18], [19]. ${ }^{2}$ On the other hand, the quantum electromagnetic field is described by a set of bosonic (Fock state) creation and annihilation operators $a^{\dagger}$ and $a$, respectively [20]. Physically, these modes are similar to the raising and lowering operators above in the sense that the two operator sets model how energy quanta are created or destroyed throughout the system's dynamical evolution. ${ }^{3}$ Each mode of the electromagnetic field is labeled by $\mathbf{k} \in \mathbb{R}^{3}$, the wavevector, and a discrete index $s \in\{1,2\}$, for the two degrees of photon polarization [14]. We abbreviate this multimodal index by the notation $\kappa:=(\mathbf{k}, s)$ and hereafter write $a_{\kappa}^{\dagger}$ and $a_{\kappa}$ for the creation and annihilation operators, respectively, associated with the $\kappa$ th bosonic radiation mode. The pure (non-interacting) Hamiltonians of the QD and the field are $H_{0}^{\mathrm{QD}}$ and $H_{0}^{\mathrm{rad}}$, respectively.

The total Hamiltonian operator $H$ of the coupled $\mathrm{Q} /$ field system can be written in the following form:

$$
H=\underbrace{H_{0}^{\mathrm{QD}}+H_{0}^{\mathrm{rad}}+H_{0}^{\mathrm{int}}}_{H_{0}}+\underbrace{H^{\mathrm{int}}(t)}_{\text {external interaction }}
$$

Here, $H_{0}$ is the time-independent Hamiltonian of the coupled system, while $H^{\text {int }}(t)$ is the time-dependent interaction Hamiltonian. The latter term is solely due to interaction with external (most often classical) current source. In this paper this external source can be taken as a classical applied electric field, for example laser field or electrode (battery) field. On the other hand, note that the time-independent Hamiltonian $H_{0}$ does contain coupling or interaction terms, namely the term $H_{0}^{\text {int }}$, which models the (time-independent) interaction between the radiation field modes and the various QDs's energy levels.

In order to see this clearly, we give the complete operator expressions for all these terms in the total Hamiltonian (1). We start with the self-interaction (diagonal) terms

$$
H_{0}^{\mathrm{QD}}=\sum_{i=1}^{M_{\mathrm{d}}} \hbar \omega_{i} \sigma_{i}^{+} \sigma_{i}^{-}, \quad H_{0}^{\mathrm{rad}}=\sum_{\kappa=1}^{M_{\mathrm{m}}} \hbar \omega_{\kappa} a_{\kappa}^{\dagger} a_{\kappa},
$$

for the free (self interaction) parts of the $\mathrm{QD}$ array, $H_{0}^{\mathrm{QD}}$, and the radiation $H_{0}^{\mathrm{rad}}$ field. Here, $M_{\mathrm{m}}$ is the number of radiation modes taken into consideration, while $\hbar$ is the reduced Planck constant. The (radian) energy transition frequency of the $i$ th QD (there are $N_{\mathrm{d}}$ of them) is $\omega_{i}$, while the corresponding frequency for the $\kappa$ th radiation field mode is $\omega_{\kappa}$.

\footnotetext{
${ }^{2}$ The reverse operation $\sigma_{i}^{-}\left|\varepsilon_{2}^{i}\right\rangle=\left|\varepsilon_{1}^{i}\right\rangle$ applies to the lowering operator. We always have $\sigma_{i}^{-}\left|\varepsilon_{1}^{i}\right\rangle=\sigma_{i}^{+}\left|\varepsilon_{2}^{i}\right\rangle=0$.

${ }^{3}$ The bosonic oscillators, however, are not localized in space like QDs, so the spacetime quantum field linked to $a^{\dagger}$ and $a$ can carry energy and information to distant locations [20].
}

The total interaction Hamiltonian $H_{0}^{\text {int }}$ can be decomposed into two components as follows:

$$
H_{0}^{\text {int }}=H_{0}^{\text {dot } / \text { field }}+H_{0}^{\text {dot } / \text { dot }}
$$

The coupling between the QDs and the radiation modes is modeled using the following interaction Hamiltonian:

$$
H_{0}^{\mathrm{dot} / \text { field }}=\sum_{i=1}^{M_{\mathrm{d}}} \sum_{\kappa=1}^{M_{\mathrm{m}}} \gamma_{\kappa i} a_{\kappa}^{\dagger} \sigma_{i}^{-}+\gamma_{\kappa i}^{*} a_{\kappa} \sigma_{i}^{+} .
$$

Here, the generally complex numbers $\gamma_{\kappa i} \in \mathbb{C}$ are the entries of the coupling matrix of the QD/field system. Physically, the number $\gamma_{\kappa i}$ measures the quantum energy exchange between the $i$ th QD and the $\kappa$ th radiation field mode where this exchange is due to coupling interaction between the two modes. ${ }^{4}$ The interaction form (4) is the rotating wave approximation (RWA) where terms in the Hamiltonian having the form $a_{\kappa} \sigma^{-}$ or $a_{\kappa}^{\dagger} \sigma^{+}$are neglected [14]. Similarly, the dot-dot interaction Hamiltonian $H_{0}^{\text {dot/dot }}$ may also be prescribed via the RWA as follows:

$$
H_{0}^{\mathrm{dot} / \mathrm{dot}}=\sum_{i, j=1}^{M_{\mathrm{d}}} \gamma_{i j} \sigma_{i}^{+} \sigma_{j}^{-}+\gamma_{i j}^{*} \sigma_{i}^{-} \sigma_{j}^{+} .
$$

Again, the generally complex numbers $\gamma_{i j}$ quantify the energy exchange between the $i$ th and $j$ th QDs due to direct QD-QD coupling interaction. All these interaction strengths measured by the coupling matrices $\gamma_{i j}$ and $\gamma_{\kappa i}$, are generally position dependent. The dependence on position $\mathbf{x}$, however, is adsorbed into the index $i$ since each QD is located at $\mathbf{x}_{i}$ so we will not explicitly mention $\mathbf{x}$ in what follows.

We next move to the interaction between an external classical source $\mathbf{E}^{\mathrm{ex}}(\mathbf{x}, t)$ and the QDA system. We work within the electric dipole approximation, i.e., coupling occurs through an electric-dipole/electric-field type interaction of the form $-\mathbf{d}_{\mathrm{e}}^{i} \cdot \mathbf{E}^{\mathrm{ex}}(\mathbf{x}, t)$, where $\mathbf{d}_{\mathrm{e}}^{i}$ is the dipole operator of the $i$ th QD given by $\mathbf{d}_{\mathrm{e}}^{i}=\mathbf{d}_{i}^{*} \sigma^{-}+\mathbf{d}_{i} \sigma^{+}$, while the complex classical vector $\mathbf{d}_{i}:=\left\langle\varepsilon_{2}^{i}\left|\mathbf{d}_{\mathrm{e}}\right| \varepsilon_{1}^{i}\right\rangle$ is the $\varepsilon_{1} \rightarrow \varepsilon_{2}$ dipole transition matrix element between the energy levels $\varepsilon_{1}^{i}$ and $\varepsilon_{2}^{i}$ of the $i$ th qbit [14], [19], [21]. The total external field/dot interaction Hamiltonian can be written then as

$$
H^{\mathrm{int}}(t)=-\sum_{i=1}^{M_{\mathrm{d}}}\left(\mathbf{d}_{i}^{*} \sigma_{i}^{-}+\mathbf{d}_{i} \sigma_{i}^{+}\right) \cdot \mathbf{E}^{\mathrm{ex}}\left(\mathbf{x}_{i}, t\right),
$$

where use was made of the assumption that physical external classical fields are real and that the $i$ th QD is very small compared with the external field wavelength so the field at the dot can be approximated by its value at $\mathbf{x}_{i}$.

\section{Time-Domain Evolution Through the Master EQUATION METHOD}

Our goal now is to find a suitable method to compute the time evolution of a coupled QD/field system such as the QDA shown in Fig. 1. To accomplish that, we use the master equation method, which describes the temporal development

\footnotetext{
${ }^{4}$ Individual radiation energy modes $\left(a_{\kappa}^{\dagger}, a_{\kappa}\right)$ don't exchange energy for distinct values of $\kappa$ because Maxwell's equations are linear [20].
} 
of a given quantum system $\mathrm{S}$ coupled to external fluctuating quantum environments, where the latter are assumed to be large enough to stay unaffected by the evolution of the quantum system itself [17]. Here, the quantum system $\mathrm{S}$ consists of the coupled QD/field oscillators. If we have $N_{\mathrm{d}}$ QDs, each with two energy eigenstates $\varepsilon_{1}^{i}$ and $\varepsilon_{2}^{i}$, coupled to $N_{\mathrm{m}}$ radiation modes, each truncated to $N_{\mathrm{r}}$, then the combined Hilbert space of the QD/field system is the tensor product $\mathcal{H}_{S}=\mathcal{H}_{\mathrm{QD}} \otimes \mathcal{F}_{\text {rad }}$, where $\mathcal{H}_{\mathrm{QD}}$ is the QD's $2^{N_{\mathrm{d}}}$ Hilbert space, while $\mathcal{F}_{\text {rad }}$ is the $N_{\mathrm{r}}^{N_{\mathrm{m}}}$ bosonic Fock space of the QDA's quantized radiation field. Now let $|\psi\rangle \in \mathcal{H}_{\mathrm{QD}}$ be a quantum state representing an array of $N_{\mathrm{d}}$ QDs, while $|\bar{n}\rangle \in \mathcal{F}_{\text {rad }}$ is the array of occupation (number) Fock states representing the quantum state of the radiation field of $N_{\mathrm{m}}$ modes. A general quantum state $|\Psi\rangle$ of the coupled QD/field system is then the product vector $|\Psi\rangle=|\psi\rangle|\bar{n}\rangle \in \mathcal{H}_{S}$, where $\mathcal{H}_{S}$ is as defined above. In this paper, our analysis will be based on the statistical density operator formalism of quantum physics, which can automatically handle both pure and mixed statistical states using the operator $\rho(t)$ (sometimes written $\rho_{t}$ ) [22]. If we couple a fluctuating environment $E$ to the system $\mathrm{S}$, the combination $\mathrm{S}+\mathrm{E}$ may be treated as a single unit described by a pure state $\left|\Psi_{\mathrm{S}+\mathrm{E}}\right\rangle$, or a density operator $\rho_{\mathrm{S}+\mathrm{E}}$ (pure or mixed state), each evolving according to either the Schrodinger or von Neumann dynamic law of quantum theory, respectively [14], [22], [23]. However, since we are interested only in the dynamics of S, which is the QDA in our case, we average out all degrees of freedom pertinent to $\mathrm{E}$ by defining a reduced density operator through the partial trace operation $\rho(t):=\rho_{\mathrm{S}}(t)=\operatorname{Tr}_{\mathrm{E}}\left\{\rho_{\mathrm{S} \times \mathrm{E}}(t)\right\}$, where $\rho_{\mathrm{S}}(t)$ is the reduced density operator of the system $\mathrm{S}$ whose time dynamics is under consideration [17].

The time evolution of $\rho(t)$ is governed by a special (but general enough for our purposes) master equation known as the Gorini-Kassakowski-Sudarshan-Lindblad (GKSL) equation [15], [24]. The master equation is written in terms of a special superoperator $\mathcal{L}$, where it takes the form $\mathrm{d} \rho(t) / \mathrm{d} t=$ $\mathcal{L} \rho(t)$, i.e., that of a first-order differential equation in the infinite-dimensional Liouville space (superspace of quantum operators [25]). The formal solution of the evolution law can be expressed as $\rho(t)=\exp (\mathcal{L} t) \rho(0)$, which is a Markov semigroup. Therefore, $\mathcal{L}$ plays the role of the infinitesimal generator of stochastic evolution in the open dynamics of quantum systems. ${ }^{5}$ Under some general but standard assumptions, ${ }^{6}$ it can be shown that the following master equation is the most general one that can lead to trace-preserving and completely positive evolution of the density operator ${ }^{7}$ [15], [24]:

$$
\frac{\mathrm{d} \rho}{\mathrm{d} t}=\frac{-\mathrm{i}}{\hbar}\left[H, \rho_{t}\right]+\sum_{j} \Gamma_{j}\left(L_{j} \rho_{t} L_{j}^{\dagger}-\frac{1}{2}\left\{L_{j}^{\dagger} L_{j}, \rho_{t}\right\}\right) .
$$

\footnotetext{
${ }^{5} \mathrm{~A}$ concrete example of $\mathcal{L}$ is given by the GSKL equation (7), whose examination is immediately given below.

${ }^{6}$ Namely the following: 1) the initial system-environment composite is uncorrelated, 2) the relaxation time of the environment is much shorter than the system, and 3) the dynamics is local in time (Markovianity assumption) [17].

${ }^{7}$ Both of these conditions on the evolution of $\rho(t)$ are required for the consistency of quantum mechanics. For the definition of a completely positive operator, see [15], [17].
}

The anticommutator is defined by $\{a, b\}:=a b+b a$. The numbers $\Gamma_{j} \in \mathbb{R}^{+}$characterize the relaxation rates of the various quantum dissipation/decoherence processes captured by the corresponding jump operators $L_{j}$. The later physically model how the system $\mathrm{S}$, evolving under its own Hamiltonian $H$, interacts with the external environment $\mathrm{E}$ (typically an infinite reservoir). As a basic example of a quantum relaxation process, in what follows we quantitatively treat the special but important and commonly encountered practical case of quantum dissipation processes. Here, each physical degree of freedom labeled by $j$ can be assigned a jump operator $L_{j}=a$ or $L_{j}=\sigma^{-}$, depending on whether we have a material or bosonic oscillator. The relaxation rate $\Gamma_{j}=1 / T_{j}$ will be specified in terms of the relaxation time $T_{j}$. Other open quantum system operations such as dephasing (decoherence) can be easily modeled as well. Note that for an $M$-dimensional open quantum system, the total number of independent quantum jump operations in the GKSL equation (7) cannot exceed $M^{2}-1$.

\section{Quantum Antenna Directivity: Construction AND NUMERICAL RESULTS}

We now put together all of the ingredients of the basic quantum model of the QDA system developed above in order to explore the possibility of analyzing a concrete radiating quantum dot array excited by external laser field. The basic configuration is shown in Fig. 1, composed of two QDs, labeled as $\mathrm{QD}_{1}$ and $\mathrm{QD}_{2}$, which are allowed to directly couple to each other, for example via quantum tunneling. Let the number operator of the $\kappa$ th mode be $N_{\kappa}=a_{\kappa}^{\dagger} a_{\kappa}$. We define the time-domain directivity of an open quantum antenna system, described by the reduced density operator $\rho(t)$, along a solid angular sector $\Omega$, by the expression:

$$
D(\Omega, t):=\frac{4 \pi}{\Omega} \frac{\sum_{\kappa \in \Omega} \hbar \omega_{\kappa} \operatorname{Tr}\left\{N_{\kappa} \rho(t)\right\}}{\sum_{\kappa \in 4 \pi} \hbar \omega_{\kappa} \operatorname{Tr}\left\{N_{\kappa} \rho(t)\right\}},
$$

where $\operatorname{Tr}$ is the operator trace. In this expression, directions in space are labeled by the mode index $\kappa$, where we have deployed a recent idea of constructing the radiation pattern of antennas in complex media using the momentum space formulation [26]-[29]. Physically, the expression (8) proposes to define the directivity of a QDA along a given angular sector $\Omega$ as the ratio of the angular energy density radiated by the dominant photon field modes, whose momenta (wavevectors) are directed along $\Omega$, to the isotropic total radiation energy. ${ }^{8}$

The radiation field is composed of an infinite number (continuum) of modes indexed by $\kappa=(\mathbf{k}, s)$. However, in practical calculation only a finite number of modes can be dealt with in a discretized numerical simulation. In what follows, we then consider the scenario depicted in Fig. 1 where for simplicity only three modes are considered, each with a wavenumber $\mathbf{k}$ directed into one specific direction in space. While in the exact theory should be continuous, the directivity may then be approximated by a discrete model easier to investigate in a preliminary analysis.

\footnotetext{
${ }^{8}$ Recall that the isotropic density is the total energy (in all modes, i.e., along all directions) divided by the total solid angle $4 \pi$ [9].
} 


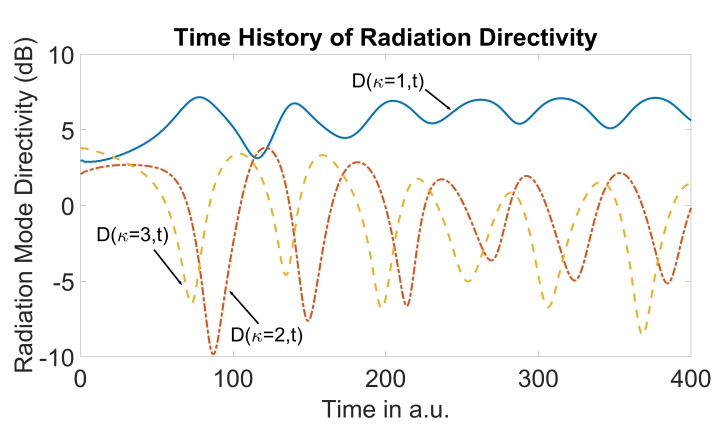

Fig. 2: QDA directivity time history calculations.

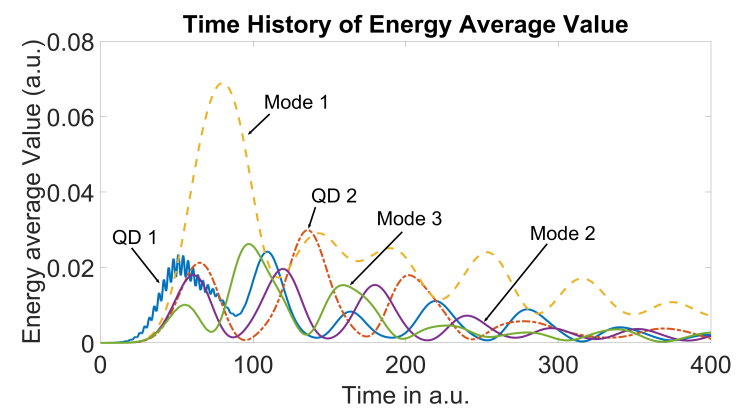

Fig. 3: Total energy of QDs and radiations modes as functions of time evolution. $\mathrm{QD}_{1}$ is coupled to an external laser's Gaussian pulse field.

Note that the the factor $\hbar \omega_{\kappa}$ appearing in (8) is the radiation field photon (oscillator) energy. On the other hand, the input excitation field is assumed to be a modulated Gaussian pulse of the form $E^{\mathrm{ex}}(\mathbf{x}, t)=E^{\mathrm{ex}}(\mathbf{x}) g(t)$, where $E^{\mathrm{ex}}(\mathbf{x})$ is a unit point basis function in the sense that $E^{\mathrm{ex}}\left(\mathbf{x}_{i}\right)=$ $1, i=1,2, \ldots, N_{\mathrm{d}}$, while the time waveform is given by $g(t)=A_{\mathrm{e}} \exp \left[-\left(t-t_{\mathrm{d}}\right)^{2} / t_{\mathrm{p}}^{2}\right] \cos \omega_{\mathrm{e}}\left(t-t_{\mathrm{d}}\right)$, where in what follows we set the pulse amplitude to $A_{\mathrm{e}}=1$, the delay time $t_{\mathrm{d}}=50$, pulsewidth $t_{\mathrm{p}}=30$, and the modulation frequency $\omega_{e}=1$ all in atomic units. ${ }^{9}$ In this manner the strength of the external field coupling to the $i$ th dot is completely controlled by $A_{\mathrm{e}}$ and the dipole moment $\mathbf{d}_{i}$, while the frequency of the driving field is $\omega_{\mathrm{e}}{ }^{10} \mathrm{We}$ set $N_{\mathrm{r}}=5$ (five possible photons per mode) and choose $\omega_{\kappa}=1$ for all values of $\kappa=1,2,3$. The two QDs's energy gaps $\omega_{\mathrm{i}}=\varepsilon_{2}^{i}-\varepsilon_{1}^{i}, i=1,2$, are equal and identical to $\omega_{\kappa}=1$ (resonant condition). Only $\mathrm{QD}_{1}$ is coupled to the Gaussian external electric field defined above. However, we allow a mutual interaction between $\mathrm{QD}_{1}$ and $\mathrm{QD}_{2}$. Table I shows optimized values for the QDA's coupling matrix $\gamma_{\kappa i}$ obtained through a random search algorithm (more elaborate optimization methods will be used in the future). The dipole matrix element for the external field $\mathbf{E}^{\mathrm{ex}} / \mathrm{QD}$ coupling is chosen as 0.04 a.u.. In addition, we inserted two jump operators into (7) in order to include dissipation processes in both quantum dots, with lifetime $\Gamma_{i}$ set equal to 50 atomic time units.

\footnotetext{
${ }^{9}$ Throughout this paper, atomic units are denoted by a.u., where we set $\hbar=c=1$.

${ }^{10}$ Recall we assume that the spatial variations of the external field are ignored throughout any QD so the field spatial function on the QD domain is just unity.
}

\begin{tabular}{cc}
\hline \hline Radiation mode $(\kappa) /$ Quantum dot $(i)$ & $\gamma_{\kappa i}$ (a.u.) \\
\hline$\kappa=1, i=1$ & 0.050 \\
$\kappa=1, i=2$ & 0.0013 \\
$\kappa=2, i=1$ & 0.045 \\
$\kappa=2, i=2$ & $0.045 \exp (-\mathrm{i} \pi / 6)$ \\
$\kappa=3, i=1$ & 0.0055 \\
$\kappa=2, i=2$ & 0.055 \\
$i=1, i=2$ & 0.050 \\
\hline \hline
\end{tabular}

TABLE I: Optimized QDA coupling matrix $\gamma_{\kappa i}$ used to generate the results in Figs. 3 and 2.

The resulting system has a combined Hilbert space of dimension $M=500$. Thus, the corresponding GKSL master equation (7) for this example consists of a 250,000 coupled ordinary differential equations. (This incredible increase in size is a direct feature of all open dynamical systems approach.) A 4th-order Runge-Kutta method [30] was implemented to perform the numerical integration of the above coupled system of differential equations. (The accuracy of the integration was checked against benchmark quantum physics examples with known analytical solutions such as the qbit system evolution under external fields and the Jane-Cummings model [14].) The simulation time step resolution is 0.1 in a.u. time units with maximum number of 400 iterations. The time-domain directivity pattern itself is given in Fig. 2 . We can clearly see that mode $1(\kappa=1)$, which corresponds photons going into the $\theta=\pi / 3$ direction (see Fig. 1) is noticeably larger than the other two modes directivities. This was acheived with the use of the optimized values in Table I. On the other hand, if the phase of $\gamma_{\kappa=2, i=1}$, i.e., the coupling between mode 2 and $\mathrm{QD}_{1}$, was set to zero, then the three values of $D(\kappa, t)$ are found to have roughly the same time average level, indicating no directive behaviour. In other words, introducing relative phase excitation can lead to significant enhancement in the directive properties of the QDA array, a situation well known in classical antenna theory but is shown here to $b$ valid also in the quantum case. Finally, Fig. 3 shows the evolution of the average energy of all modes and QDs operators. We can see that mode 1 is dominant in the early time in terms of the total energy. However, the directivity calculations of Fig. 2 shows that mode 1 continues to dominate (relatively) for late time too. This difference illustrates the importance of the directivity formula (8) as a new measure for characterizing energy dynamics in quantum systems.

\section{CONCLUSION}

We introduced a general computational method suitable for the analysis and design of generic dissipative quantum dot array antenna systems. The algorithm can model mutual coupling between QDs and the interaction of an arbitrary classical external source field with the system. The evolution of the density matrix of the QDA system is implemented by setting up and numerically integrating the GSKL master equation, effectively treating the problem as a stochastic dynamic system. An example was given illustrating the analysis and the design of a two-level 2-element QD array excited by a Gaussian laser field. The directivity of the quantum 
radiation field was computed and found to exhibit a steering capacity depending on the relative phase excitations of the individual QDs. The proposed method can be used to analyze and optimize future quantum antenna transmitters for quantum wireless communications.

\section{REFERENCES}

[1] S. Imre and F. Azs, "Quantum computing and communications an engineering approach," 012005.

[2] G. Benenti, G. Casati, D. Rossini, and G. Strini, Principles of quantum computation and information: a comprehensive textbook. Singapore Hackensack, NJ: World Scientific Publishing Co. Pte. Ltd, 2019.

[3] L. Novotny, Principles of Nano-Optics. Cambridge: Cambridge University Press, 2012.

[4] A. A. Arisheh, S. Mikki, and N. Dib, "A subwavelength-laser-driven transmitting optical nanoantenna for wireless communications," IEEE Journal on Multiscale and Multiphysics Computational Techniques, vol. 5, pp. 144-154, 2020.

[5] G. Y. Slepyan, S. Vlasenko, and D. Mogilevtsev, "Quantum antennas," Advanced Quantum Technologies, vol. 3, no. 4, p. 1900120, Apr. 2020

[6] S. Mikki, "Quantum antenna theory," in 2017 IEEE AP-S Symposium on Antennas and Propagation and USNC-URSI Radio Science Meeting July 9-14, 2017 San Diego, California, USA. IEEE Antennas \& Propagation Society, 2017.

[7] S. Mikki, "Quantum antenna theory for secure wireless communications," in 2020 14th European Conference on Antennas and Propagation (EuCAP), 2020, pp. 1-4.

[8] S. A. Schelkunoff and H. T. Friss, Antennas: Theory and practice. New York; Chapman \& Hall: London, 1952

[9] C. A. Balanis, Antenna Theory: Analysis and Design, 4th ed. Interscience: Wiley, 2015.

[10] S. Mikki, "A quantum MIMO architecture for antenna wireless digital communications," Progress In Electromagnetics Research C, vol. 93, pp. 143-156, 2019.

[11] S. Mikki and M. Herde, "Analysis and design of secure quantum communication systems utilizing electromagnetic Schrodinger coherent states," Quantum Engineering, vol. 3, no. 3, 2021.

[12] G. Y. Slepyan and A. Boag, "Quantum nonreciprocity of nanoscale antenna arrays in timed Dicke states," Phys. Rev. Lett., vol. 111, p. 023602, Jul 2013.

[13] T. Ihn, Semiconductor nanostructures: quantum states and electronic transport. Oxford New York: Oxford University Press, 2010.

[14] J. C. Garrison and R. Chiao, Quantum optics. Oxford: Oxford University Press, 2014.

[15] V. Gorini, A. Kossakowski, and E. C. G. Sudarshan, "Completely positive dynamical semigroups of N level systems," Journal of Mathematical Physics, vol. 17, pp. 821-825, 1976.

[16] Chang, Quantum stochastics. New York: Cambridge University Press, 2014.

[17] H.-P. Breuer and F. Petruccione, The theory of open quantum systems. Oxford New York: Oxford University Press, 2002.

[18] W. Louisell, Radiation and noise in quantum electronics. Huntington, N.Y: R.E. Krieger Pub. Co, 1977.

[19] L. Mandel and E. Wolf, Optical coherence and quantum optics. Cambridge: Cambridge University Press, 1995.

[20] J. Klauder and E. C. G. Sudarshan, Fundamentals of quantum optics. Mineola, N.Y: Dover Publications, 2006.

[21] P. Milonni, An introduction to quantum optics and quantum fluctuations. Oxford, United Kingdom: Oxford University Press, 2019.

[22] P. A. M. Dirac, The principles of quantum mechanics. Oxford England: Clarendon Press, 1981.

[23] J. Neumann, Mathematical foundations of quantum mechanics. Princeton: Princeton University Press, 2018.

[24] G. Lindblad, "On the generators of quantum dynamical semigroups," Communications in Mathematical Physics, vol. 48, no. 2, pp. 119-130, Jun 1976.

[25] I. Prigogine, Non-Equilibrium Statistical Mechanics. Mineola: Dover Publications, 2017.

[26] S. Mikki, "Exact derivation of the radiation law of antennas embedded into generic nonlocal metamaterials: A momentum-space approach," in 2020 14th European Conference on Antennas and Propagation (EuCAP), 2020, pp. 1-5.
[27] S. Mikki, "Theory of electromagnetic radiation in nonlocal metamaterials - Part I: Foundations," Progress In Electromagnetics Research B, vol. 89, pp. 63-86, 2020.

[28] - "Theory of electromagnetic radiation in nonlocal metamaterials Part II: Applications," Progress In Electromagnetics Research B, vol. 89, pp. 87-109, 2020.

[29] — , "Proca metamaterials, massive electromagnetism, and spatial dispersion," Annalen der Physik, vol. 533, no. 8, p. 2000625, June 2021.

[30] D. F. Griffiths and D. J. Higham, Numerical methods for ordinary differential equations: initial value problems. London New York: Springer, 2010. 